7 Flaherty KR, Mumford JA, Murray S, et al. Prognostic implications of physiologic and radiographic changes in idiopathic interstitial pneumonia. Am J Respir Crit Care Med 2003; 168: 543-548.

8 Raghu G, Brown KK, Costabel U, et al. Treatment of idiopathic pulmonary fibrosis with etanercept: an exploratory, placebo-controlled trial. Am J Respir Crit Care Med 2008; 178: 948-955.
9 Taniguchi H, Ebina M, Kondoh Y, et al. Pirfenidone in idiopathic pulmonary fibrosis. Eur Respir J 2010; 35: 821-829.

10 Antoniou KM, Hansell DM, Rubens MB, et al. Idiopathic pulmonary fibrosis: outcome in relation to smoking status. Am J Respir Crit Care Med 2008; 177: 190-194.

\title{
Obstructive lung function in sarcoidosis may be missed, especially in older white patients
}

\section{To the Editors:}

Lung function abnormalities are often present when patients are first diagnosed with pulmonary sarcoidosis, and most studies to date indicate that the predominant pattern is of restriction or normal airways, with a truly obstructive pattern in a minority [1]. The largest case-control study of sarcoidosis to date, the ACCESS (A Case Control Etiologic Study of Sarcoidosis) trial, showed that the biggest group of patients in that particular cohort $(46.9 \%)$ presented with a forced expiratory volume in $1 \mathrm{~s}$ (FEV1)/forced vital capacity (FVC) ratio of $>80 \%$ and only $13.2 \%$ presented with a ratio of $50-69 \%$ [2]. Of the 215 patients from the ACCESS trial who were followed up for a further 2 yrs, the majority showed no change in pulmonary function, radiographic stage or dyspnoea score [3]. A more recent European study showed that patients with sarcoidosis had impaired lung compliance and a reduced diffusing capacity of the lung for carbon monoxide (DL,CO) and, while there was no predominance of restrictive lung defects, airway obstruction was only present in $11.7 \%$ of patients [4].

Over the past 3 yrs, we have noticed that an increasing number of patients who present to our clinics in West London, UK with a new diagnosis of pulmonary sarcoidosis have a predominantly obstructive lung defect. A detailed analysis of lung function in sarcoidosis with respect to stratification by patient demographics has, to our knowledge, not been previously reported. We present the results of a systematic retrospective analysis of 164 consecutive patients presenting with a new diagnosis of pulmonary sarcoidosis over a 10-yr period and in whom formal lung function was recorded at the time of presentation. All patients had a clinically definite diagnosis, with supportive histology where available, and the subsequent follow-up examinations were in keeping with the original diagnosis. Lung function testing was carried out using a standardised protocol (single-breath method for gas transfer and nitrogen washout for lung volumes) and recorded electronically. All testing was performed at a single institution and statistical comparisons were performed by MannWhitney U-tests and multiple linear regression analysis.

Our data show that the percentage of patients presenting with an obstructive lung defect of FEV1/FVC ratio 50-69\% was higher than expected, at $23.2 \%$ (fig. 1a), while $42.1 \%$ of patients presented with FEV1/FVC $>80 \%$. There was no significant difference in smoking status (the majority were nonsmokers), diagnosis of asthma or chronic obstructive pulmonary disease (COPD) between the obstructive and restrictive groups. We also found that patients with an obstructive defect (median $53.5 \mathrm{yrs}$, interquartile range (IQR) 39-61.25 yrs) were significantly older $(p=0.0015)$ than those with restrictive defects (median 44 yrs, IQR 33-50.5 yrs) (fig. 1b).

A significant difference $(p=0.028)$ was found in lung function between the main ethnic groups, with black patients having a higher FEV1/FVC ratio (median 81\%, IQR 73-85\%) than white patients (median 78\%, IQR 69-83\%). In addition, the proportions of lung function pattern within ethnicities was dramatically different (fig. 1c). White patients $(n=83)$ presented with a restrictive/obstructive ratio of 1.4 , while this ratio was more than twice as large in black patients $(n=54)$, at 4.0. The ratio of Asian (Indian subcontinent) patients $(n=27)$ was similar to that in the white population, at 1.3. There was no difference found between the ethnicities in terms of other measured lung function parameters, including the $\mathrm{DL}, \mathrm{CO} \%$ predicted (fig. 1d), residual volume or FVC.

On multivariate analysis of the black patient group (online supplementary table 1), there were no significant demographic or clinical associations found, suggesting that the greater proportion of black patients seen presenting with a restrictive lung pattern in this cohort was not due to factors such as presence of co-existing lung disease or demographic differences (e.g. 5.6\% of black patients and $6 \%$ of white patients were recorded as having asthma or COPD at the time of diagnosis of sarcoidosis). Only a small number of patients with obstructive lung defects were assessed for bronchodilator response, but in those who were $(n=9)$, we saw no significant improvement in lung function (mean change in FEV1 5\%, range -8-13\%).

Almost one in four of all patients in this cohort presented with an FEV1/FVC ratio of $50-69 \%$ and this obstructive pattern of lung function was significantly more common in those who were older and who were white. One possible explanation for our findings of a larger than expected group with significant airway obstruction may be our routine use of endobronchial ultrasound sampling for lymph node disease. This relatively specialised method, with greater diagnostic sensitivity in sarcoidosis [5], may be providing a histological confirmation in patients in whom a diagnosis of 

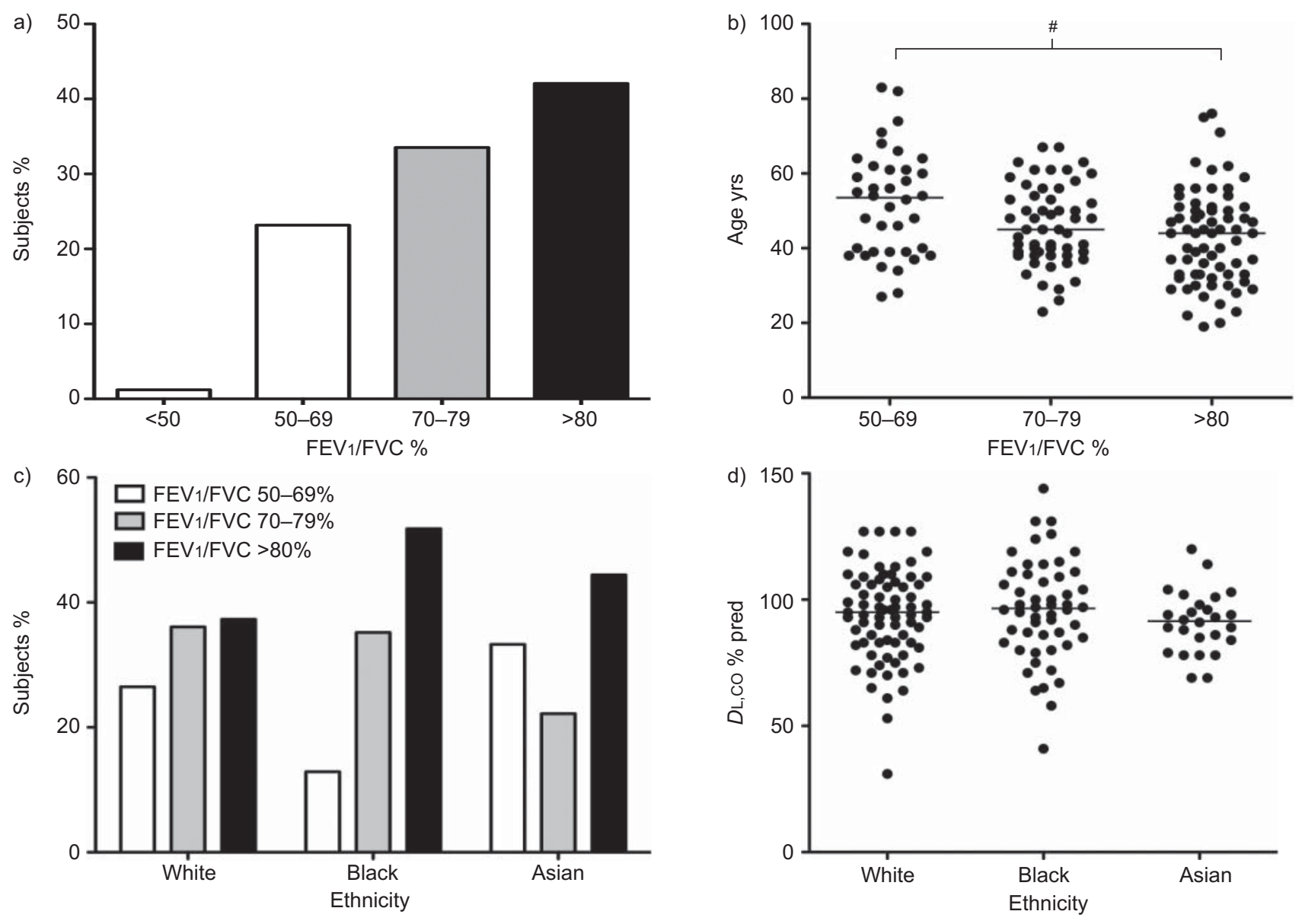

FIGURE 1. a) In this cohort, $23.2 \%$ of patients with pulmonary sarcoidosis presented with a forced expiratory volume in $1 \mathrm{~s}$ (FEV1)/forced vital capacity (FVC) ratio of $50-$ $69 \%$. b) The median age of patients with obstructive lung disease at presentation was significantly higher (53.5 yrs) than that of those with restrictive disease (44 yrs). c) The ratio of restrictive/obstructive lung function at first presentation differed within ethnic groups, with black patients displaying a higher ratio (4.0) than white patients (1.4). d) Other measures of lung function did not differ between ethnic groups, including diffusing capacity of the lung for carbon monoxide $(D L, C O) \%$ predicted $(\%$ pred), residual volume and FVC. ${ }^{*}: p=0.0015$.

obstructive airway disease may otherwise have been made. Indeed, there are numerous published case reports of patients with obstructive bullous lung disease in whom the underlying diagnosis of sarcoidosis has been originally missed [6].

These data are the first to stratify lung function in sarcoidosis by patient demographics, but we cannot fully explain the significantly higher median age at presentation found in patients with obstructive disease. There is some evidence to suggest that the FEV1 reduces as individuals get older, by as much as $6-8 \%$ per decade [7], and an FEV1 that declines at a faster rate than the FVC might help explain our findings in part, but is unlikely to represent the entire difference seen.

The different ratios of restrictive/obstructive disease seen between ethnic groups may be partly explained by ethnic differences in baseline lung function in healthy populations [7], but our data are contrary to the only previously published work in this area, which showed that the predominant airway disease in a cohort of African-Americans with sarcoidosis was one of obstruction, rather than restriction [8]. Our findings may in part be due to differences in ethnic lineage, with a greater percentage of descendants from the West Indies making up the UK black population compared to that seen in the USA [9].

The clinical course of sarcoidosis is often more severe in black patients [10] and the recent definition of clinical phenotypes by the World Association of Sarcoidosis and Other Granulomatous Diseases showed that African-American patients were likely to have a higher clinical outcome status, indicating more chronic disease [11]. Less than $10 \%$ of the patients classified as black in the present study had self-declared as being of mixed race, which should not, therefore, have affected the outcome and our findings of a higher incidence of restrictive airways disease in black European patients need to be investigated further. Notably, accurate chest radiographic staging was not available for all patients, but we do not feel that this has affected the interpretation of our results, as there was no association found between the presence of lung fibrosis and black ethnicity on multivariate analysis (online supplementary table 1). Additionally, chest radiographic staging may be interpreted differently, even between expert radiologists, and there is no evidence to date for a relationship between airway obstruction and radiographic stage in sarcoidosis [8]. 
We believe that our study is the first to identify different proportions of obstructive and restrictive airway disease between the main ethnic groups in sarcoidosis, and also the first to report an association of functional impairment with age. Despite a weak evidence base, sarcoidosis patients are often prescribed inhaled corticosteroids and $\beta$-agonists for respiratory symptoms regardless of their pattern of lung function at presentation [12]. Large airway obstruction in sarcoidosis is associated with increased mortality [13] and our data suggest that these patients may represent an under-diagnosed group. Further studies are needed to confirm these demographic associations, as well as to assess disease progression and response to inhaled therapies in this particular clinical phenotype of sarcoidosis.

\section{Thillai*, L. Potiphar*, C. Eberhardt*, M. Pareek*, R. Dhawan ${ }^{\#}$, O.M. Kon ${ }^{\#}$, M. Wickremasinghe", A. Wells ${ }^{\natural}$, D. Mitchell ${ }^{\top}$ and A. Lalvani*}

*National Heart and Lung Institute, Imperial College London, "St Mary's Hospital, and 'Interstitial Lung Unit, Imperial College London NHS Healthcare Trust, London, UK.

Correspondence: M. Thillai, Wright-Fleming Institute, National Heart and Lung Institute, Imperial College London, Praed Street, London W2 1NY, UK. E-mail: m.thillai@imperial.ac.uk

Statement of Interest: None declared.

Acknowledgements: We would like to thank S. Martin and the lung function team at St Mary's Hospital (Imperial College London NHS Healthcare Trust, London, UK) for their assistance with data collection.

\section{REFERENCES}

1 Handa T, Nagai S, Fushimi Y, et al. Clinical and radiographic indices associated with airflow limitation in patients with sarcoidosis. Chest 2006; 130: 1851-1856.
2 Baughman RP, Teirstein AS, Judson MA, et al. Clinical characteristics of patients in a case control study of sarcoidosis. Am J Respir Crit Care Med 2001; 164: 1885-1889.

3 Judson MA, Baughman RP, Thompson BW, et al. Two year prognosis of sarcoidosis: the ACCESS experience. Sarcoidosis Vasc Diffuse Lung Dis 2003; 20: 204-211.

4 Boros PW, Enright PL, Quanjer PH, et al. Impaired lung compliance and $\mathrm{DL}, \mathrm{CO}$ but no restrictive ventilatory defect in sarcoidosis. Eur Respir J 2010; 36: 1315-1322.

5 Tremblay A, Stather DR, Maceachern P, et al. A randomized controlled trial of standard $v s$ endobronchial ultrasonographyguided transbronchial needle aspiration in patients with suspected sarcoidosis. Chest 2009; 136: 340-346.

6 Jeebun V, Forrest IA. Sarcoidosis: an underrecognised cause for bullous lung disease? Eur Respir J 2009; 34: 999-1001.

7 Hankinson JL, Odencrantz JR, Fedan KB. Spirometric reference values from a sample of the general U.S. population. Am J Respir Crit Care Med 1999; 159: 179-187.

8 Sharma OP, Johnson R. Airway obstruction in sarcoidosis. A study of 123 nonsmoking black American patients with sarcoidosis. Chest 1988; 94: 343-346.

9 Agyemang C, Bhopal R, Bruijnzeels M. Negro, Black, Black African, African Caribbean, African American or what? Labelling African origin populations in the health arena in the 21st century. J Epidemiol Community Health 2005; 59: 1014-1018.

10 Baughman RP, Teirstein AS, Judson MA, et al. Clinical characteristics of patients in a case control study of sarcoidosis. Am J Respir Crit Care Med 2001; 164: 1885-1889.

11 Baughman RP, Nagia S, Balter M, et al. Defining the clinical outcome status (COS) in sarcoidosis: results of WASOG Task Force. Sarcoidosis Vasc Diffuse Lung Dis 2011; 28: 56-64.

12 Alberts C, van der Mark TW, Jansen HM. Inhaled budesonide in pulmonary sarcoidosis: a double-blind, placebo-controlled study. Dutch Study Group on Pulmonary Sarcoidosis. Eur Respir J 1995; 8: 682-688.

13 Viskum K, Vestbo J. Vital prognosis in intrathoracic sarcoidosis with special reference to pulmonary function and radiological stage. Eur Respir J 1993; 6: 349-353.

\section{Reduced GM-CSF autoantibody in improved lung of autoimmune pulmonary alveolar proteinosis}

\section{To the Editors:}

Pulmonary alveolar proteinosis (PAP) is a rare lung disease characterised by excessive accumulation of surfactant materials within alveolar spaces [1]. Patients with autoimmune PAP (aPAP) present a high level of granulocyte-macrophage colonystimulating factor (GM-CSF) autoantibodies (GM-Ab) in the serum as well as in bronchoalveolar lavage fluid (BALF) [2]. GM$\mathrm{Ab}$ neutralise the biological activity of GM-CSF in the lung [3], impairing terminal differentiation of alveolar macrophages and macrophage-mediated pulmonary surfactant clearance [4].

Based on the aetiology, clinical trials of exogenous GM-CSF supplementation have been carried out by a number of physicians with variable response rates ranging from 40 to $62 \%$ [5-9]. Previously, we reported that in three patients who received a pilot GM-CSF inhalation therapy, oxygenation was improved and the concentration of GM-Ab in BALF was reduced [7]. BONFIELD et al. [8] also reported that the serum titre of GM-Ab was reduced during successful treatment of aPAP with subcutaneously injected GM-CSF. However, our recent phase II trial of GM-CSF inhalation involving 35 patients revealed that serum levels of $\mathrm{GM}-\mathrm{Ab}$ remained unchanged throughout the therapy, suggesting that GM-CSF inhalation therapy did not affect the production of GM-Ab [9]. Thus, the effect of exogenous GM-CSF administration on GM-Ab levels in the serum remains controversial. This discrepancy may be due to differences in the route 